\title{
Pre-Socratic Discrete Kinematics
}

\author{
Claudio Calosi and Vincenzo Fano \\ University of Urbino
}

DOI: $10.2478 /$ disp-2013-0002

BIBLID [0873-626X (2013) 35; pp. 21-31]

\begin{abstract}
We present a neglected heterodox version of Zeno's paradox of the Stadium, underlining some problems that a discrete kinematics would have to account for. Building on our reconstruction of the Stadium argument we provide new arguments to show that a discrete kinematics cannot uphold three independently plausible assumptions about motion, that we label No Switching, Granular Continuity and Different Velocities, and hence it should drop at least one.
\end{abstract}

\section{Keywords}

Zeno's Paradoxes, Discreteness, Space and Time, Motion, Velocity.

There exists a somewhat heterodox and neglected version of Zeno's paradox of the Stadium, which was first presented by Tannery (1885: 394) and then developed by Evellin 1893. This version was supposed to overcome the apparently evident flaw in the argument presented in Aristotle's reconstruction of the paradox in Phys. 239b 33 - 240a 19. Indeed, this flaw was thought to have been much too great a mistake for Zeno, the very inventor of dialectics according to Diogenes Laertius, not to have noticed ${ }^{1}$. In the first half of the twentieth century this heterodox version received a great deal of attention, whereas nowadays it is thought to be ungrounded due to a lack of textual evidence. However, despite its historical merit, or lack thereof, it has a certain theoretical value of its own.

Here is a slightly revised account of such a version. Let $\left(x_{1}, x_{2}, x_{3}\right)$ and $\left(y_{1}, y_{2}, y_{3}\right)$ be two sets of equal masses displaced in such a way that, at time $t_{1}, x_{1}$ is vertically aligned with $y_{1}, x_{2}$ with $y_{2}$, and $x_{3}$ with

${ }^{1}$ See Davey 2007.

Disputatio, Vol. V, No. 35, May 2013

Received: 01/06/2012. Revised: 03/10/2012. Accepted: 05/11/2012 
$y_{3}$ (Fig. 1a). Suppose that both space and time are discrete. Let us call an atomic unit of time an "instant" and an atomic unit of space a "region". Now suppose that all the $x$ masses move to the left with velocity $v_{x}$ of one region per instant and that the $y$ masses move to the right with a velocity $v_{y}$ of the same magnitude. After an instant, at $t_{2}$, $y_{1}$ will be vertically aligned with $x_{3}$ (Fig. 1b). Zeno concludes that this is paradoxical, for there has to be an intervening instant between $t_{1}$ and $t_{2}$ at which $y_{1}$ is vertically aligned with $x_{2}$

Evellin (1893: 385-387), Russell (1903: 352) and Whitrow (1961: 136-137) argued that Zeno's argument fails because to require the existence of an intervening instant between $t_{1}$ and $t_{2}$ is illicit, as it amounts to reintroducing if not the continuity, at least the denseness of time, which is ruled out by the discreteness assumption.

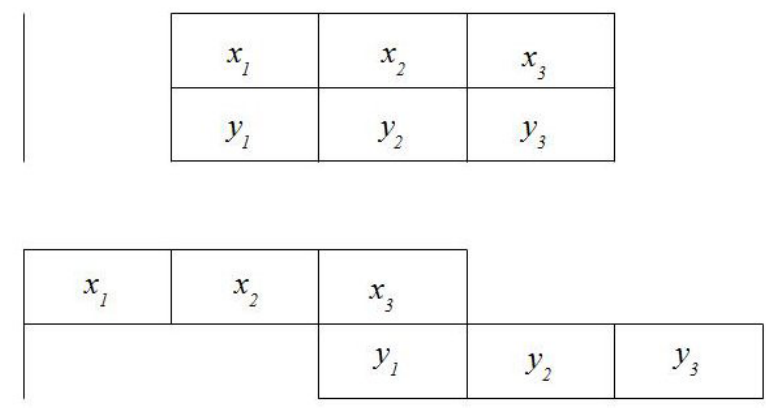

(a)

Fig. 1a, 1b.

According to their reading, if space and time really are discrete, $y_{1}$ is never aligned with $x_{2}$. Hugget 2010 explains the point by suggesting that it is better to think of quantized space as a matrix of lights that holds some pattern of illuminated lights for each instant, rather than a chessboard where each piece is frozen in one particular region at one particular instant. If so, he urges, we should not be misled into thinking that the lights on in some regions at $t_{1}$ move to other regions at $t_{2}$.

Grünbaum (1968: 118-120) points out that the vertical alignment 
of $y_{1}$ and $x_{2}$ is in fact possible depending on the relative velocity of the $x$ s and $y s$. For example let the $x$ and $y$ masses be displaced in the same way they were at $t_{1}$ (Fig. 1a); but now let the $x$ masses be at rest ${ }^{2}$ and let the $y$ masses move with velocity $v_{y}$ of one instant per region towards the right. In this case, at $t_{2}, y_{1}$ is indeed vertically aligned with $x_{2}$ (Fig. 1c).

\begin{tabular}{|l|l|l|l|}
\hline$x_{1}$ & $x_{2}$ & $x_{3}$ & \multicolumn{1}{|}{} \\
\hline & $y_{1}$ & $y_{2}$ & $y_{3}$ \\
\hline
\end{tabular}

Fig. 1c.

Let us stipulate that a happening or a fact ${ }^{3} e_{1}$ is actual iff there exists an instant $t$ at which $e_{1}$ takes place. Moreover two happenings $e_{1}$, $e_{2}$ are coactual iff there is an instant $t$ at which they are both actual. So, in the first case we presented, the facts that $y_{1}$ occupies $R_{y}$ and $x_{2}$ occupies $R_{x}$, where $R_{y}$ and $R_{x}$ are vertically aligned, are not coactual $^{4}$ and thus, the alignment of $y_{1}$ with $x_{2}$ is not actual, i.e. does not have event status in Grünbaum's terminology, whereas in the second case it is. Thus, whether a vertical alignment qualifies as actual at all depends upon the magnitude of the relative velocity between the two sets of masses. Grünbaum then calls our attention to this consequence of the argument that reveals a kinematic problem for discrete space and time. Let us quote him directly:

'This consequence has the significance of a caveat for the following reason: as far as I am aware, none of our present-day kinematic knowledge even gives a hint of the possibility of the aforementioned dependence of event-status [coactuality] on relative motion'. (Grünbaum 1968: 119-

\footnotetext{
${ }^{2}$ With respect to a particular rest frame, which, in the original case, is the stadium.

${ }^{3}$ We are using these terms very broadly.

${ }^{4}$ For the very simple reason that each of them is never actual in our terminol-
} ogy. 
120 , italics in the original)

First of all let us notice that, as it stands, Grünbaum's remark seems quite puzzling. There seem to be in fact lots of $\operatorname{cases}^{5}$ in which there is some dependence of event-status on relative motion. Suppose two masses, Achilles and the turtle, are moving on a straight line along the same direction, say to the right, and suppose furthermore that they are located at different points on the line, Achilles being to the left of the turtle. Then, whether Achilles will catch up with the turtle, i.e. whether the catching up of the turtle by Achilles has an event status in Grünbaum's terminology ${ }^{6}$, will depend on their relative motion. Let $V_{A}$ and $V_{t}$ be their velocities. If $V_{A}=V_{t}$, Achilles will never catch up with the turtle and their spatial separation will be unchanged. If $V_{A}>V_{t}$, Achilles will eventually catch up with the turtle (pace Zeno), and if $V_{A}<V_{t}$ not only Achilles will never catch up with the turtle but their spatial separation will increase. Therefore it seems that Grünbaum's observation is not correct after all, at least when taken at face value.

These considerations notwithstanding there is something deeply relevant about Grünbaum's recognition of the important role of relative motion in a discrete kinematics. He was the first one to acknowledge that the possibility of relative motion raises a metaphysical problem for discrete kinematics. He took this problem to be the dependence of event status on relative velocity. We already argued that this is not, at first sight, a problem after all. But this does not mean that the original observation about the tension between relative motion and discrete space and time was off the track. In the rest of the paper we will argue that this tension reveals that the conjunction of three independently very plausible ${ }^{7}$ assumptions about motion is inconsistent ${ }^{8}$. Hence a discrete kinematics should drop at least one

${ }^{5}$ At first sight this holds both for continuous and discrete space and times. We will see that the situation is slightly more complicated when space and time are discrete.

${ }^{6}$ Or it is actual in the terminology we have introduced.

${ }^{7}$ We will return on the issue of the plausibility later on.

${ }^{8}$ This is something similar in its logical structure to Diodorus' so called master argument. 
of them. Let us label these assumptions No Switching (NS), Granular Continuity (GC) and Different Velocities (DV) respectively.

Before giving a rough formulation of the assumptions, let us note that in a discrete kinematics there cannot be particles smaller than a region, for, if it were the case, the locations of such particles would have to be subregions of the alleged region. And this is impossible because regions are supposed to be atomic'.

Here is our formulation of the assumptions:

(NS) Let $x$ and $y$ be two impenetrable ${ }^{10}$ particles moving on the same straight ${ }^{11}$ line in opposite directions. Then they cannot switch their position;

(GC) Let $x$ be a particle and let $\left(R_{1}, R_{2}, \ldots, R_{n}\right)$ be $n$ distinct adjacent regions such that there is no missing region in between ${ }^{12}$. Then if $x$ moves from $R_{1}$ to $R_{n}$ it has to pass through each $R_{i}$ in between $R_{1}, R_{n}^{13}$

$(D V)$ Each and every particle can have different velocities.

Let us spend a few words on these assumptions. Their initial plausibility seems to stem out from simply looking at the world around us. Many forms of matter ${ }^{14}$ seem to be characterized by impenetrability. Hence they cannot switch positions without clashing. Just think of two trains on the same railroad track. Also, walk from the desk in your office to the door following a straight line (don't cheat, don't jump). You would have passed through all the spatial regions in be-

${ }^{9}$ Thus, from now on, when we say that a particle is located at a region, we mean that it has the same size, shape and dimensions of the region.

${ }^{10}$ Also, we are assuming that two distinct particles cannot be located at the same region at the same instant. Whether this follows from impenetrability already or it has to be assumed independently is controversial.

${ }^{11}$ That is, the particles move along the same geodetic and the metric structure does not change over time.

${ }^{12}$ Our formulation is inspired by White (1992: 273).

${ }^{13}$ We take this to mean it has to occupy all the regions, the ith region after the i-1th region in subsequent instants.

${ }^{14}$ Not so for photons. 
tween the desk and the door. Finally, things seem to move at different velocities. It will not take the same time to get to your office if you're walking rather than driving. Moreover note that if $D V$ does not hold, two distinct particles could not have different velocities.

We want to argue that a discrete kinematics cannot have them all. First we argue that $G C$ entails not $D V$, i.e.:

(1) $G C \rightarrow \sim D V$

Our argument for (1) is the following. Let us call the velocity $=1$ region per instant $V_{a}$ for "allowed velocity". Then suppose (1) does not hold. There could be two cases, either there is some velocity $V<V_{a}$ or there is some velocity $V_{+}>V_{a}$. In either case, given discrete space and time, there should be a number $k \in \mathrm{N}^{15}$ such that:

(2) $V_{-/+}=k V_{a}$

But clearly there is no such natural number $k$ that solves equation (2) and such that $V<V_{a}$. This argument establishes that there cannot be any velocity that is smaller than what we called the "allowed velocity". Can there be a greater one? Then at each instant the alleged material particle would have crossed a spatial distance of $k$ regions. But, given the discreteness assumption, there could not be any instant at which the particle would have passed through the $k$ parts of that distance. GC entails that the particle has passed ${ }^{16}$ through each of the $k$ parts of that distance, yet it is not even reasonable to ask when it has passed there, given $D V$. This argument seems to establish that there is no possible velocity that is greater than the "allowed velocity" $V_{a}$. This yields, together with the previous argument, that (1) does indeed hold. A-fortiori GC entails that two distinct particles cannot have different velocities.

Next, we want to argue that $D V$ entails not NS, i.e.:

(3) $D V \rightarrow \sim N S$

We argue in favor of (3) by contraposition. First we show that NS entails that all particles have velocity $=1$ region per instant, i.e. what

\footnotetext{
${ }^{15}$ The fact that $k$ is a natural number follows from the discreteness assumption.

${ }^{16}$ Though it has never exactly occupied any of them.
} 
we labeled $V_{a}$ in the previous argument.

Suppose that it is not so. Let $x$ and $y$ be two particles and let $R$, $R$ and $R$ be three regions such that they are adjacent, $R$ lying between $R_{x}^{y}$ and $R_{y}$ and $R_{x}$ being the first region on the left (Fig. 2a). Suppose now that at instant $t_{1}, x$ occupies $R_{x}$ and $y$ occupies $R_{y}$. Let $x$ move with velocity $v_{x}>V_{a}$, for instance $v_{x}=2$ regions per instant towards the right, and let $y$, instead, move with $v_{y}=2$ regions per instant towards the left. It follows that at instant $t$, we will have the following displacement: $x$ will occupy $R_{y}$ and $y$ will occupy $R_{x}$, i.e. $x$ and $y$, the two particles, will have switched their positions (Fig. 2b). This is because it is never actual that $x$ has occupied $R$ for there is no intervening instant between $t_{1}$ and $t_{2}$. The same goes for $y$. And the "banging and bouncing" of $x$ and $y$ is thus never actual, as this could only have taken place at $R$, in the way we have set things up. And we have just argued that it is never actual that either $x$ or $y$ occupy $R$. We have already argued that there cannot be a velocity ${ }_{V}<V_{a}$, so this argument establishes that NS entails $\sim D V$. Claim (3) now follows straightforwardly by contraposition.

It seems that the intuition $D V$ brings about in a discrete framework is that motion is a sort of appearing/disappearing (perhaps at non adjacent regions) phenomenon. Thus it should not be surprising that it does not seat well with either GC or NS.

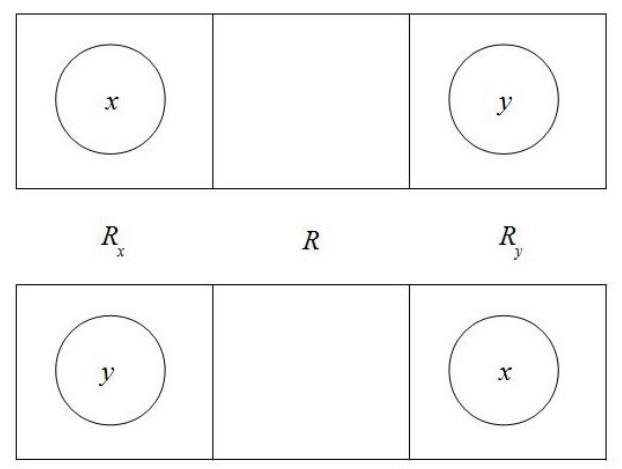

(a)

(b)

Fig. 2a, 2b. 
It might occur to someone to block this argument invoking exactly $G C$, for $G C$ would ensure that $x$ and $y$ will have to pass through $R$ whatever their velocities are, thus having a chance of colliding after all. This objection is however mistaken. This is simply because by simple contraposition (1) will yield:

(4) $D V \rightarrow \sim G C$

So that you cannot invoke $G C$ in presence of $D V$. Now, claims (1) and (3) together entail that a discrete kinematics cannot uphold all of the assumptions we started with. That their conjunction is inconsistent is easily seen for we have that:

(5) $N S \wedge G C \wedge D V \rightarrow \sim D V$,

by (1), and that:

(6) $N S \wedge G C \wedge D V \rightarrow \sim N S$,

by (3).

Our arguments raise a natural question. Even if it is not possible to have all the three assumptions, is it possible to have at least two of them?

On the one hand claim (1) and its converse, establish that it is not possible to have both $G C$ and $D V$. On the other hand claim (3) and its converse establish that it is not possible to have both $D V$ and NS. This leaves open only one possibility, namely that of retaining both NS and GC.

But finally, we want to argue that

(7) $G C \rightarrow \sim N S$

Thus leaving with a three-fold exclusion. The argument for $(7)^{17}$ goes roughly as follows. Take two particles $x$ and $y$ moving in opposite directions with velocity ${ }^{18} V_{a}$ along a series of adjacent regions $R_{1}, \ldots, R_{n}$. Given $G C$ they will have to pass through each region in the series. Then there will be an instant $t_{1}$ such that the particles will be exactly located at two adjacent regions in the series at $t_{1}$. And it follows from the velocities they have that at the next instant $t_{2}$ the two

${ }^{17}$ This argument was suggested to us by an anonymous referee of this journal.

18 This follows from our argument in favor of claim (1). 
particles will have switched their positions ${ }^{19}$.

From claims (1), (3), (4), (7) and their converses it then follows:

(8) $N S \rightarrow \sim G C ; N S \rightarrow \sim D V$

$$
\begin{aligned}
& \mathrm{GC} \rightarrow \sim \mathrm{NS} ; \mathrm{GC} \rightarrow \sim \mathrm{DV} \\
& \mathrm{DV} \rightarrow \sim \mathrm{NS} ; \mathrm{DV} \rightarrow \sim \mathrm{GC}
\end{aligned}
$$

That is, the endorsement of one of the assumptions we started with entails that we should drop the other two. Now, which way to go?

Note that NS is violated upon very weak conditions. In fact even if the argument for (7) mentions explicitly $G C$, it does not need to do so. Given GC, and its entailment of $V_{a}$ as the only permissible velocity, we are guaranteed that there will be an instant in which the particles will find themselves at adjacent regions. However we just need to add this possibility independently of $G C$ and the argument would still go through. So, all that it takes for NS to be violated is the possibility of moving particles at adjacent regions at the same instant.

$G C$, as plausible as it might seem, entails that there is only one allowed velocity. But we seem to have an overwhelming body of experiences in favor of the contrary. Things seem to move at different velocities around us.

And in fact, $D V$ seems a difficult assumption to drop. There are indeed weird ways in which $D V$ could be salvaged on the face of our arguments. Sorabji (1983: 384) for example points out ${ }^{20}$ that a particle may linger for several instants in the same region and then move

${ }^{19}$ There is a possible reply to this argument, and it is to contend that, given impenetrability, which we have assumed in our formulation of NS, the particles will not switch their position at $t_{2}$ but rather remain where they were at $t_{1}$. This way of resisting the argument however rests upon a very strong reading of impenetrability, namely one that rules out the possibility of switching between adjacent positions. But this reading will render NS utterly unproblematic. And then its incompatibility with $D V$ will be difficult to see. On a weaker reading of impenetrability, one that only precludes spatial overlapping, it becomes compatible with switching between adjacent positions, and the argument goes through. We are indebted to an anonymous referee of this journal for having pointed out to us these possibilities.

${ }^{20}$ Sorabji attributes this solution in turn to an Arabic atomist of the IX century, Abū l-Hudhayl al-'Allāf. 
on. Its average velocity could then be smaller than what we label "allowed velocity" ${ }^{21}$. But these attempts of salvaging $D V$ sound extremely ad hoc. We should not need to resort to them. We did not intend these remarks as exhausting the pros and cons of retaining and dropping different assumptions, but rather as suggesting different possibilities open to further investigations.

Following Grünbaum's lead on the tension between relative motion and discrete kinematics we have shown that within that framework it is not possible to uphold different assumptions about motion that seem at first sight overwhelmingly plausible. But this plausibility probably stems out from the endorsement of a paradigm of continuity for motion established already in Aristotle's physics.

However different programs in contemporary physics attempt at quantizing spacetime (if not space and time) thus endorsing discreteness. One of the most promising is the so called Loop Quantum Gravity. However, according to one of its main proponents, namely Carlo Rovelli, 'this discreteness of geometry $[\ldots]$ is very different from the naïve idea that the world is made by discrete bits of something' (Rovelli 2001: 110).

It thus remains to be assessed how such programs would resolve the sort of "metaphysical trilemma" we have envisaged for our Pre-Socratic discrete kinematics. It could very well be the case that it does not even arise in those contexts. In this case the trilemma would not be solved but rather dissolved. And then it would have to be assessed whether some other dilemmas are lurking. ${ }^{22}$

Claudio Calosi University of Urbino, Department of Foundations of Science Via Timoteo Viti 10, 61029 Urbino, Italy claudio.calosi@uniurb.it

\footnotetext{
${ }^{21}$ This proposal raises interesting questions. We could push the point that the arguments go through only for the average velocity of the particle. Its instantaneous velocity would still be constrained to be $=V_{a}$. However in this proposal there is no guarantee that particle trajectories would turn out to be differentiable, so that the usual notion of instantaneous velocity defined as the first derivative of the position function would not be applicable.

${ }^{22}$ We would like to thank an anonymous referee for extremely helpful comments and insightful suggestions.
} 
Vincenzo Fano University of Urbino, Department of Foundations of Science Via Timoteo Viti 10, 61029 Urbino, Italy vincenzo.fano@unurb.it

\section{References}

Davey, Kevin. 2007. Aristotle, Zeno and the stadium paradox. History of Philosophy Quarterly 24: 127-148.

Evellin, François. 1893. Le mouvement et les partisans des indivisibles. Revue de Métaphysique et de Morale 1: 382-95.

Grünbaum, Adolf. 1968. Modern Science and Zeno's Paradoxes. London: Allen and Unwin.

Hugget, Nick. 2010. Zeno's paradoxes. At: http://plato.stanford.edu/entries/ paradox-zeno/\#Sta

Rovelli, Carlo. 2001. Quantum Spacetime: What Do We Know? In Philosophy Meets Physics at Planck Scale, ed. by C. Callender and N. Hugget. Cambridge: Cambridge University Press.

Russell, Bertrand. 1903. Principles of Mathematics. London: Allen and Unwin.

Sorabji, Richard. 1983. Time, Creation and the Continuum. Chicago: University of Chicago Press.

Tannery, Paul. 1885. Le concept scientifique du continu. Zénon d'Elée et Georg Cantor. Revue Philosophique 20: 385-410.

White, Michael. 1992. The Continuous and the Discrete. Ancient Physical Theories from a Contemporary Perspective. Oxford: Clarendon Press.

Whitrow, Gerald James. 1961. The Natural Philosophy of Time. London: Nelson. 